\title{
RECTIFIABILITY IN TEICHMÜLLER THEORY
}

\author{
KARI ASTALA and MICHEL ZINSMEISTER \\ Université Bordeaux I, CNRS, Mathématiques \\ 351 Cours de la Libération, F-33405 Talence, France
}

1. The universal Teichmüller space. A simply connected domain $\Omega$ of the plane is well parametrized by its Riemman mapping, i.e. a conformal mapping from the unit disk $\Delta$ onto $\Omega$. The idea of universal Teichmüller space (UTS for short) is to equip the set of all those conformal mappings with an (infinite dimensional) complex structure, in order to study deformations of such domains.

In order to guess what is this complex structure, let us first define a holomorphic deformation of the unit disk as a family $f_{\lambda}, \lambda \in \Delta$ of conformal mappings $\Delta \rightarrow \mathbb{C}$ (i.e. holomorphic and injective) such that:

(i) $f_{0}(z)=z$,

(ii) $\forall z \in \Delta, \lambda \mapsto f_{\lambda}(z)$ is holomorphic in $\Delta$.

The complex structure on UTS should be of course such that, when restricted to a holomorphic deformation of the disk it coincides with the $\lambda$-structure. Now, by the Koebe distortion theorem, for every Riemann mapping $f$ on the unit disk, the function $\log f^{\prime}$ belongs to the Banach space $\mathcal{B}$ of holomorphic functions $b$ in $\Delta$ satisfying

$$
\|b\|_{\mathcal{B}}=\sup _{z \in \Delta}(1-|z|)\left|b^{\prime}(z)\right|
$$

(more precisely, this is a complex Banach space of functions modulo constants, which is natural in this setting - we identify two domains if they are similar). The function $\lambda \mapsto \log \left(f_{\lambda}^{\prime}\right)$ is holomorphic from $\Delta$ to $\mathcal{B}$ and sends 0 to 0 ; by the Schwarz lemma, we must have

$$
\left\|\log f_{\lambda}^{\prime}\right\|_{\mathcal{B}} \leq|\lambda| .
$$

Conversely, Nehari's univalence criterion implies that a function $b \in \mathcal{B}$ with sufficiently small norm has to be of the form $b=\log f^{\prime}$ for some conformal

1991 Mathematics Subject Classification: 30F60, 30D60.

The paper is in final form and no version of it will be published elsewhere. 
mapping $f$; moreover there exists a holomorphic deformation such that $f=f_{\lambda}$ with $|\lambda| \sim\left\|\log f^{\prime}\right\|_{\mathcal{B}}$. Thus the analytic structure has to be the structure of the complex Banach space $\mathcal{B}$. We then define

$$
S=\left\{\log f^{\prime} ; f \text { is conformal from } \Delta \text { to } \mathbb{C}\right\},
$$

the universal Teichmüller space $T(I)$ being the interior of $S$ in $\mathcal{B}$.

The UTS is closely related to the theory of quasiconformal mappings; a homeomorphism of the plane is said to be quasiconformal if it belongs to the Sobolev space $W_{2, \text { loc }}^{1}(\mathbb{C})$ and if there exists a function $\mu \in L^{\infty}(\mathbb{C}),\|\mu\|_{\infty}<1$ such that

$$
\frac{\partial f}{\partial \bar{z}}=\mu \frac{\partial f}{\partial z}
$$

Ahlfors and Bers [1] have shown that $\log f^{\prime} \in T(I)$ iff $f$ admits a quasiconformal extension to the plane; the corresponding domains $f(\Delta)$ are thus the Jordan domains called quasidisks whose boundary is characterized by Ahlfors' three points condition

$$
\exists C>0 ; \forall z_{1}, z_{2}, z_{3} \text { in this order on } \partial \Omega, \quad\left|z_{1}-z_{2}\right| \leq C\left|z_{1}-z_{3}\right| .
$$

The deepest result in this theory is a corollary of the Riemann mesurable theorem which asserts that given $\mu \in L^{\infty}(\mathbb{C}),\|\mu\|_{\infty}<1$, there exists an (essentially unique) solution of (1) which is quasiconformal in the plane. This theorem has a long history: In this (very general) setting, it is due to Bojarski [2]. The geometrical corollary of this theorem is that the UTS $T(I)$ is contractible; every quasidisk can thus be continuously (in the $\mathcal{B}$ topology) deformed into a disk.

We finish this section with a last geometric property of the UTS: if $\left(f_{\lambda}\right)$ is a holomorphic deformation of the unit disk then $\log f_{\lambda}^{\prime} \in T(I)$ for every $\lambda$ in the disk. This property, due to Mañé, Sad and Sullivan [3], is called the $\lambda$-lemma.

2. Deformation of rectifiable curves. This theory allows us to study how certain quantities attached to quasidisks vary when we deform those quasidisks; for instance, how varies the Hausdorff dimension of a quasicircle? A theorem of Ruelle asserts that this variation is real-analytic in some holomorphic disks of the UTS. We will return to this point in a later section.

The object of which we would like to study now the variations is an operator, namely the Cauchy integral operator, the operator of $L^{2}(d s)$ defined on smooth curves ( $d s$ is arclength measure) by

$$
C f(z(s))=P . V \cdot \int_{0}^{L} \frac{f(z(t))}{z(s)-z(t)} d z(t),
$$

where P.V. stands for principal value, $z(s)$ is arclength parametrization, and $\mathrm{L}$ is the length of the curve.

The UTS is obviously not well suited for this study since a domain has to be rectifiable in order to give at least a meaning to this operator; but any neighborhood of 0 in $T(I)$ contains domains with fractal boundary. 
We thus need a different Teichmüller theory to handle this problem. We begin with a result of David [4]: if $\Gamma$ is a rectifiable curve then the operator defined by (3) is continuous on $L^{2}(\Gamma, d s)$ if and only if it satisfies the following, known as Ahlfors-David regularity:

$$
\exists C>0 ; \forall z \in \Gamma, \forall r>0, \quad \operatorname{length}(\Gamma \cap D(z, r)) \leq C r .
$$

In [5], it is proved that if the simply connected domain $\Omega$ has an Ahlfors-David regular boundary, then, if $f: \Delta \rightarrow \Omega$ is the Riemann mapping, Log $f^{\prime}$ belongs to the space $B M O A(D)$ of functions $f \in H^{2}(\Delta)$ such that

$$
\exists C>0 ; \forall I \text { interval } \subset \partial \Delta, \frac{1}{|I|} \int_{I}\left|f(z)-\left(\frac{1}{|I|} \int_{I} f(u)|d u|\right)\right|^{2}|d u| \leq C .
$$

Moreover, if $\left(f_{\lambda}\right)$ is a holomorphic deformation of the unit disk such that $f_{\lambda}(\partial \Delta)$ is AD-regular for every $\lambda \in \Delta$ then, by the $\lambda$-lemma $f_{\lambda}(\Delta)$ is also a quasidisk for $\lambda \in \Delta$ and it can be shown very easily that the curves satisfying both Ahlfors regularity condition and the 3 -points condition are exactly the Lavrentiev curves, i.e. the curves $\Gamma$ satisfying

$$
\exists C>0 ; \forall z, \zeta \in \Gamma, \quad \operatorname{length}(\gamma(z, \zeta)) \leq C|z-\zeta|,
$$

where $\gamma(z, \zeta)$ is the smallest subarc of $\Gamma$ with extremities $z, \zeta$. A theorem of Pommerenke asserts that $f(\Delta)$ is a Lavrentiev domain if $\log f^{\prime}$ has a small norm in $B M O A(\Delta)$.

The right analogue of the UTS appears then to be

$$
\mathcal{L}=\left\{\log f^{\prime} \in S ; f(\Delta) \text { is a Lavrentiev domain }\right\},
$$

the ambient space being here the complex Banach space $B M O A(\Delta)$. In [5] it is proved that $\mathcal{L}$ is the interior in $B M O A(\Delta)$ of

$$
\mathcal{R}=\left\{\log f^{\prime} \in S ; \partial f(\Delta) \text { is AD-regular }\right\} .
$$

Next we recall the fundamental result of Tukia: The Lavrentiev domains are exactly the images of the unit disk under bilipschitz homeomorphisms of the plane, i.e. homeomorphisms $\Phi$ for which there exists $C>0$ such that:

$$
\forall z, \zeta \in \mathbb{C}, \quad C^{-1}|z-\zeta| \leq|\Phi(z)-\Phi(\zeta)| \leq C|z-\zeta|
$$

This shows a very nice parallel between the two theories; the UTS is the space associated to the geometry of quasiconformal mappings, while $\mathcal{L}$ deals with the bilipschitz geometry. Unfortunately the parallel has a limit; first of all, the set $\mathcal{R}$ is not a good analogue of $S$ because it is not closed in $B M O A(\Delta)$; secondly there is no (known) analogue of the Riemann mesurable theorem in the bilipschitz case: It is not known if $\mathcal{L}$ is contractible, or even if it is connected.

Certainly completely new tools are to be invented to prove (or disprove) that $\mathcal{L}$ is contractible. Before this theory exists, we modestly present here a compromise between the two theories which yields to (we hope) interesting new results. 
Before doing this let us conclude this section as it began; in [5] it is shown that the Cauchy operator varies analytically in $\mathcal{L}$.

3. Universal Teichmüller space and BMOA. The compromise between the two theories merely consists in defining

$$
\Sigma=S \cap B M O A(\Delta)
$$

and $\mathcal{T}(I)$ as the interior of $\Sigma$ in $B M O A(\Delta)$, or, equivalently, as $T(I) \cap B M O A(\Delta)$. If $g$ is a continuous fonction $\mathbb{R} \rightarrow \mathbb{R}$ and $\Omega=\{y>g(x)\}$, then the Riemann mapping $f$ from $\Delta$ onto $\Omega$ satisfies $\log f^{\prime} \in B M O A(\Delta)$ (this follows easily from the fact that $\operatorname{Arg} f^{\prime}$ is bounded) and thus $\log f^{\prime} \in \Sigma$ does not imply that $\partial f(\Delta)$ is rectifiable. The principal drawback of this theory is thus that the space comprises non-rectifiable curves but, as we shall see, the domains under consideration are not so far from being rectifiable.

Anyhow, the lost is counterbalanced by a very nice parallel with the classical theory of the UTS.

Before we present it, let us recall the notion of conformal welding: If $\Omega$ is a quasidisk in the plane and if $\varphi, \psi$ are the conformal mappings from $\Delta$ to $\Omega$ and ${ }^{c} \bar{\Delta}$ to ${ }^{c} \bar{\Omega}$ respectively, the conformal welding associated to $\Omega$ is then defined as $h=$ $\psi^{-1} \circ \varphi$; it is a homeomorphism of $\partial \Delta$ and by results of Ahlfors, Bers, Beurling, this homeomorphism satisfies the following condition, known as quasisymmetry (we shall say that $h \in \mathcal{Q}(\mathbb{T})$ ):

$$
\exists C>0 ; \forall z \in \partial \Delta, \forall \omega \in(-\pi, \pi), \quad\left|h\left(z e^{i \omega}\right)-h(z)\right| \leq C\left|h(z)-h\left(z e^{-i \omega}\right)\right| .
$$

The importance of this concept lies in the fact that conversely for every $h \in$ $\mathcal{Q}(\mathbb{T})$ there is a unique (up to Möbius transformation) quasidisk whose welding is precisely $h$. The set $\mathcal{Q}(\mathbb{T})$ can thus be seen as a way of parametrizing $T(I)$.

It will appear that $\mathcal{T}(I)$ can be similarly parametrized; but while the elements of $\mathcal{Q}(\mathbb{T})$ may be wildly singular, the weldings corresponding to $\mathcal{T}(I)$ are very strongly absolutely continuous. More precisely let us define a strongly quasisymmetric homeomorphism of $\partial \Delta$ (this terminology is due to Semmes [6] and the set will be denoted by $\mathcal{S} \mathcal{Q}(\mathbb{T})$ ) as a homeomorphism $h$ such that

$\forall \epsilon>0, \exists \delta>0 ; \forall I$ interval $\subset \partial \Delta, \forall E \subset I$ mesurable,

$$
|E| \leq \delta|I| \Rightarrow|h(E)| \leq \epsilon|h(I)| \text {. }
$$

Before stating the main theorem, we must introduce the concept which will be crucial for our purposes; if $\Omega$ is a domain of the plane, a positive measure $\nu$ defined in $\Omega$ is called a Carleson measure if it satisfies

$$
\exists C>0 ; \forall z \in \partial \Omega, \forall r>0, \quad \nu(\Omega \cap D(z, r)) \leq C r .
$$

Let us now consider an element $\log f^{\prime}$ of $T(I)$ and denote by $h$ the corresponding welding. The main result is the following 
THEOREM 1. The following are equivalent:

(1) $\log f^{\prime} \in \mathcal{T}(I)$,

(2) $h \in \mathcal{S Q}(\mathbb{T})$,

(3) $f$ admits a quasiconformal extension to $\mathbb{C}$ with dilatation $\mu$ satisfying

$$
\frac{|\mu|^{2}}{(|z|-1)} d x d y \text { is a Carleson measure in }{ }^{c} \bar{\Delta} \text {. }
$$

(For the proof, we refer to [7]).

The domains satisfying one of the equivalent conditions of the theorem have been completely characterized by Bishop and Jones. They are the domains satisfying:

(BJ) $\exists C>0 ; \forall z \in \Omega \exists \Omega_{z} \subset \Omega$ "centered" at $z, \quad l\left(\partial \Omega_{z} \cap \partial \Omega\right) \geq C \operatorname{dist}(z, \partial \Omega)$.

In this statement, $\Omega_{z}$ is a Lavrentiev domain with constant $\leq C$ and "centered" at $z$ means that $0.01 \leq d\left(z, \partial \Omega_{z}\right) / \operatorname{diam}\left(\Omega_{z}\right) \leq 100$.

An interesting corollary of this characterization is that the class $\mathcal{T}(I)$ is invariant under bilipschitz mappings. Let us also notice that in a neighborhood of the disk, the three conditions are equivalent to being a Lavrentiev domain with small constant.

This theorem is part of a long story which started in a paper by Carleson [8] giving a sufficient condition on the dilatation of a quasiconformal self-mapping of the disk to have an absolutely continuous boundary value. Later Dahlberg [9] found the right Carleson measure condition which was generalized by Fefferman, Kenig and Pipher [10] who proved (in different terms) $(3) \Rightarrow(2)$. The Carleson measure condition in (3) has been first introduced in this context by Semmes [6] who proved $(3) \Rightarrow(2)$ in the case of small norm; his method is completely different and uses operator theory.

This theorem also shows that the problem of finding conditions either on the welding or on the dilatation of the extension equivalent to rectifiability is much harder. Actually it puts some light on the disproof by Semmes and later by Bishop of the conjecture of Jerison and Kenig [11] that welding in $\mathcal{S Q}(\mathbb{T}) \Rightarrow$ curve rectifiable; using the equivalence $(1) \Leftrightarrow(2)$, every quasidisk for which the conformal mapping $f$ satisfies $\log f^{\prime} \in B M O A(\Delta)$ is a counterexample (if not rectifiable). By a remark already made, the domain $\{y>f(x)\}$ where $f$ is the Weierstrass function

$$
f(x)=\sum_{n=0}^{\infty} \frac{\sin \left(2^{n} x\right)}{2^{n}}
$$

will do (the fact that it is a quasidisk follows from the fact that the function $f$ is in the Zygmund class).

The main corollary of theorem 1 is the result that $\mathcal{T}(I)$ is contractible as an open subset of $B M O A$. The idea to connect $\log f^{\prime} \in \mathcal{T}(I)$ to 0 is the standard one in Teichmüller theory: Using (3), we extend $f$ to be qc in the plane with dilatation 
$\mu$ satisfying the Carleson measure condition. The path is then $t \mapsto \log f_{t}^{\prime}$ where $f_{t}$ solves the Beltrami equation

$$
\bar{\partial} f_{t}=t \mu \partial f_{t} .
$$

To conclude this section we finally observe that the problem of the connectedness of the set of Lavrentiev domains $\mathcal{L}$ is still open but at least theorem 1 shows that one can connect every $\log f^{\prime} \in \mathcal{L}$ to 0 by a continuous path in $\mathcal{T}(I)$.

4. Mostow, Bowen, Ruelle. We start this section with an observation made by Poincaré more than a century ago: let $\Gamma$ be a cocompact Fuchsian group, that is, a discrete group of automorphisms of the disk without fixed point such that the quotient $\Delta / \Gamma$ is compact and let us deform the generators of $\Gamma$ into Möbius transformations in such a way that the group relations are preserved; we obtain in this way a new discrete group whose limit set is a Jordan curve (such a group is called quasifuchsian). Poincaré claimed that this limit set, if it is not a circle, must be a very "wild" Jordan curve in the sense that it should have tangents nowhere. A way (in fact the only one) to produce such deformations is by geometry; let us consider two homeomorphic copies of the Riemann surface $\Delta / \Gamma$ and $\varphi$ a homeomorphism between the two surfaces; it lifts to a homeomorphism of the sphere which conjugates $\Gamma$ to such a deformation and moreover the restriction of the lifted homeomorphism to the circle is precisely the welding of the limit set of the quasifuchsian group.

There are at least two possible measurements of "wildness" of curves, all of them concerning more or less rectifiability.

The first one is by the welding; by the classical F. and M. Riesz theorem, any rectifiable Jordan curve has an absolutely continuous welding. Conversely, if the welding is singular, it can be shown that the set of points where the curve has a tangent has zero one-dimensional Hausdorff measure.

In this setting, the 2-dimensional version of Mostow rigidity theorem exactly says that in this situation, the welding of the limit set of the quasifuchsian group is either a Möbius transformation (in which case the curve is a circle) or else is totally singular. We reformulate this property by saying that a cocompact Fuchsian group is rigid.

Let us consider more generally a Fuchsian group $\Gamma$ of the first kind, i.e. such that its limit set is the whole unit circle. We can deform such a group as before except that we now restrict to quasiconformal deformations, and it makes sense to say that $\Gamma$ is rigid. Agard [12] has generalized Mostow's theorem by proving that $\Gamma$ is rigid if it is of divergence type which is equivalent to saying that the surface $\Delta / \Gamma$ has no Green function (or is of class $O_{G}$ ). Our theorem 2, which is a corollary of theorem 1 , says that this result is best possible:

TheOREM 2. Let $\Gamma$ be any Fuchsian group of convergence type; then there exists a quasifuchsian group conjugate to $\Gamma$ whose limit set is a Lavrentiev curve. In particular a group of convergence type is never rigid. 
Idea of proof. We first use the observation that if $\Gamma$ is of convergence type, then the orbit of 0 under the group is an interpolation sequence; let now $\nu$ be an $L^{\infty}$ function with norm less than one and supported in a small disk around 0 and define

$$
\mu=\sum_{\gamma \in \Gamma} t \nu \circ \gamma \frac{\bar{\gamma}^{\prime}}{\gamma^{\prime}} .
$$

This is the dilatation of a qc automorphism of the disk conjugating $\Gamma$ to a quasifuchsian group and the interpolating property of $\Gamma$ implies that the Carleson condition (3) is satisfied; if one is careful enough in the choice of $\nu$, one can be sure that the boundary value of $\mathrm{f}$ is not a Möbius transform, and we conclude by using $(3) \Rightarrow(2)$ of theorem 1 for $t$ small enough (actually we only use the small norm implication, due to Semmes).

The second way of measuring how wild is a curve is Hausdorff dimension. A theorem of Bowen [13] asserts that the dimension of the limit set of a quasifuchsian group conjugated to a cocompact Fuchsian group is $>1$ unless this limit set is a circle. The immediate parallel with the preceeding section leads to the following interesting open problem:

QUESTION. Is this property still true for groups of divergence type?

Of course theorem 2 shows that this property never holds for groups of convergence type. We nevertheless mention this new approach because of the following result of Ruelle:

If $\Gamma$ is a Fuchsian group, a holomorphic deformation of $\Gamma$ is every family $\left(\Gamma_{t}\right)$ of quasifuchsian groups together with a family $\left(\Phi_{t}\right)$ of isomorphisms from $\Gamma$ onto $\Gamma_{t}$ such that:

(i) $\Phi_{0}=\mathrm{Id}$,

(ii) $\forall \gamma \in \Gamma, t \mapsto \Phi_{t}(\gamma)$ is holomorphic in $t \in \Delta$.

Ruelle's result [14] is then that if $\left(\Gamma_{t}\right)$ is a holomorphic deformation of $\Gamma$ cocompact, then $t \mapsto$ Hausdorff dimension of $\left(\Gamma_{t}\right)$ is real-analytic in $\Delta$.

Our last result consists in exhibiting a large class of Fuchsian groups for which this property does not hold.

A Denjoy domain is the complement in $\mathbb{C}$ of a closed set $K \subset \mathbb{R}$; such a domain is said to satisfy the Carleson property if

$$
\exists \epsilon>0 ; \forall x \in K, \forall t \leq \operatorname{diam}(K), \quad|K \cap(x-t, x+t)| \geq \epsilon t,
$$

where || stands for Lebesgue measure of $\mathbb{R}$.

Suppose now that $\Omega$ is a Carleson-Denjoy domain such that $K$ is totally disconnected. Then $\Omega \simeq \Delta / \Gamma$ where $\Gamma$ is a Fuchsian group of the first kind (and of convergence type) and we have (see [15])

THEOREM 3. There exists a holomorphic deformation of such a group $\Gamma$ for which $H D\left(L\left(\Gamma_{t}\right)\right)>1$ for $t$ near 1 and $=1$ for $t$ close to 0 . A fortiori, Ruelle's property does not hold for this group. 


\section{References}

[1] L. Ahlfors and L. Bers, Riemann's mapping theorem for variable metrics, Ann. of Math. 72 (1960), 385-404.

[2] B. Bojarski, Generalized solutions of a system of differential equations of first order and elliptic type with discontinuous coefficients, Mat. Sb. 85 (1957), 451-503 (in Russian).

[3] R. Mañé, P. Sad and D. Sullivan, On the dynamics of rational maps, Ann. Sci. Ecole Norm. Sup. 16 (1983), 193-217.

[4] G. David, Opérateurs intégraux singuliers sur certaines courbes du plan complexe, ibid. 17 (1984), 157-189.

[5] M. Zinsmeister, Domaines de Lavrentiev, Publ. Math. d'Orsay, 1985.

[6] S. Semmes, Quasiconformal mappings and chord-arc curves, Trans. Amer. Math. Soc. 306 (1988), 233-263.

[7] K. Astala and M. Zinsmeister, Teichmüller spaces and BMOA, Math. Ann. 289 (1991), 613-625.

[8] L. Carleson, On mappings, conformal at the boundary, J. Analyse Math. 19 (1967), $1-13$.

[9] B. Dahlberg, On the absolute continuity of elliptic measures, Amer. J. Math. 108 (1986), 1119-1138.

[10] R. Fefferman, C. Kenig and J. Pipher, The theory of weights and the Dirichlet problem for elliptic equations, to appear.

[11] D. Jerison and C. Kenig, Boundary behavior of harmonic functions in NTA domains, Adv. in Math. 46 (1982), 80-147.

[12] S. Agard, A geometric proof of Mostow rigidity theorem for groups of divergence type, Acta Math. 151 (1983), 231-252.

[13] R. Bowen, Hausdorff dimension of quasicircles, Publ. Math. IHES.

[14] D. Ruelle, Repellers for real analytic maps, Ergodic Theory Dynamical Systems 2 (1982), 99-107.

[15] K. Astala and M. Zinsmeister, Holomorphic families of quasifuchsian groups, ibid., to appear. 